\title{
Population medicine: A step forward in closing the research gap
}

\author{
Evridiki Patelarou ${ }^{1}$
}

AFFILIATION

1 Department of Nursing, Faculty of Health Sciences, Hellenic Mediterranean University, Heraklion, Greece

CORRESPONDENCE TO

Evridiki Patelarou. Department of Nursing, Faculty of Health Sciences, Hellenic Mediterranean University, Heraklion 71410, Crete, Greece. E-mail: epatelarou@hmu.gr

Popul. Med. 2019;1(November):1
KEYWORDS

public health, health policy, epidemiology

Received: 11 April 2019, Revised: 10 July 2019,

Accepted: 22 September 2019

https://doi.org/10.18332/popmed/108431

\section{EDITORIAL}

Population health encompasses a wide range of issues, regarding disease prevention and treatment, including community, behavioural and mental health, health economics, public policy, occupational safety, gender and reproductive problems in health, and environmental health. Conclusively, public health mainly refers to the population's physical, psychological and social well-being and it is of crucial importance to ensure the improvement of all public health aspects in every community regardless of race, ethnicity, socioeconomic status and gender.

Over the past years, substantial progress has been achieved in the field of public health. In brief, many infectious diseases, such as malaria and smallpox, have been tackled with vaccination and facilitated through the supportive campaigns that have been released from time to time ${ }^{1}$. Additionally, extensive research has contributed to a better understanding of non-communicable diseases such as cancer, cardiovascular diseases, mental health and diabetes $^{2}$. Therefore, with the knowledge of best practices, more effective prevention and treatment have been applied that have led to improved disease prognosis ${ }^{3}$. Except for research on medical issues, it is imperative to assess how this obtained knowledge will eventually impact on the attitudes, beliefs, intentions and behaviours of individuals, healthcare professionals and policymakers so that healthcare delivery systems and public health can benefit. Finally, in recent years, attention has been drawn to the social determinants of health ${ }^{4}$

However, regardless of the extensive progress that has been made, there are still countless problems to be addressed and research gaps to be covered. Usually, when best or good practices are to be implemented and adopted in real life, a vast variety of factors and barriers have to be detected and faced. Also, the multivariance and the complexity of most of the issues burden the effectiveness of any improvement effort. For this reason, scientists must be encouraged to strive for more comprehensive, methodologically correct and theory-based research and interventions, and to infuse the results of each effort in the scientific community ${ }^{5}, 2019$.

When it comes to population health, one of the most significant elements that all researchers should bear in mind is the significance of understanding the population's needs and acting according to them.

To address this gap, we have created Population Medicine, an open-access double-blind peer-reviewed online journal that encompasses all aspects of population medicine, including disease prevention and treatment, research about the community, behavioural and mental health, health economics, public policy, occupational safety, gender and reproductive issues in health, and environmental health.

Population Medicine covers major diverse health issues for both communicable and non-communicable diseases. It focuses on evidence-based research to promote effective interventions and healthcare delivery systems. Additionally, it aims to inform and enhance prevention efforts, to highlight best practices regarding behavioural and medical treatments, to evaluate and improve health care policy, and finally to contribute to the improvement of the population's physical, psychological and social well-being. We welcome your submissions.

\section{REFERENCES}

1 European Centre for Disease Prevention and Control. Vaccine-preventable diseases and immunisation - core competencies. Stockholm: ECDC; 2017. doi:10/2900/86815

2 World Health Organization. Global Action Plan for the Prevention and Control of NCDs 2013-2020. www.who.int. Accessed April 9, 2019.

3 World Health Organization. European Action Plan for Strengthening Public Health Capacities and Services, WHO 
- Regional office Europe. http://www.euro.who.int/__ data/assets/pdf_file/0005/171770/RC62wd12rev1-Eng. pdf?ua=1. Accessed April 9, 2019.

4 Camargo K. Closing the gap in a generation: Health equity through action on the social determinants of health. Global Public Health. 2011;6(1):102-105. doi:10.1080/17441692.2010.514617

5 McAteer J, Di R, Fraser A, Frank J. Bridging the academic and practice/policy gap in public health: perspectives from Scotland and Canada. Journal of Public Health. 2018;41(6):632-637. doi:10.1093/pubmed/fdy127 of Potential Conflicts of Interest and none was reported. 